\title{
Importancia de la educación superior en el proyecto del envejecimiento saludable activo
}

Laura Macías Velasco

\section{Introducción. Educación para la vida}

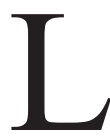

a educación del siglo xxi presenta diferentes retos entre los cuales se encuentran la urgente necesidad de vincular la escuela con el aparato productivo, los problemas sociales, el sector público y el diseño de sus programas. Un proyecto importante, que en la actualidad tienen diferentes países como tema de agenda, es el de la educación para la vida, el cual pretende que las personas tengan interés por estar en constante aprendizaje, esto con la finalidad de que su población se mantenga en la búsqueda de la mejora continua, lo cual permite tener sociedades con mejor calidad de vida y, por ello, la disminución de las problemáticas que se viven.

En este momento es apremiante considerar la educación de las personas mayores, pero no en el sentido que se ha realizado hasta el momento de "alfabetización", sino como una educación que tiene la intención de ampliar las competencias para la vida adulta, debido a que presentan diferentes circunstancias que requieren apoyo, tales como la atención en salud física y emocional, la socialización (aceptación de todos los integrantes de su familia y de la comunidad en la que viven). En este sentido, el atender las diferentes necesidades de formación de las personas mayores puede favorecer el envejecimiento saludable activo.

\section{El incremento en la población de adultos mayores}

El tema adquiere relevancia en un país que espera tener un aumento en la población de personas mayores, a consecuencia de los índices de fertilidad que se registraron en décadas anteriores, así como de la esperanza de vida que también ha aumentado (CONAPO, 2016). Además, se prevé que esa tendencia continúe, pues aunque la población de personas jóvenes ha crecido rápidamente, se espera que dicho incremento permanezca relativamente estable durante los próximos 35 años, eso quiere decir que el aumento será en porcentaje igual al actual (CONAPO, 2016), como se refiere en las cifras estadísticas que se mencionan en el siguiente párrafo para facilitar la comprensión de este fenómeno.
Desde una visión global demográfica podría pensarse que en este momento, en México, no es apremiante atender al sector de población de personas mayores, quienes representan sólo el 10.4\% de la misma, en comparación con las cifras poblaciones de Europa donde el porcentaje de adultos de 60 años y más es del $23.9 \%$, según información de la Encuesta Intercensal (INEGI, 2015). Es necesario considerar que en nuestro país, la población de 30 a 59 años pasó en esta última década de 25.5 a $36.4 \%$, dejando ver que, en el corto o mediano plazo, dicha población se integrará gradualmente al contingente de personas mayores que residen en el mismo y ello implicará contar con más servicios de atención médica, psicológica y educativa. 
Comprensión de los conceptos: persona mayor y envejecimiento saludable activo

Para iniciar con el planteamiento de la necesidad de una propuesta educativa para las personas mayores es necesario hacer una revisión de dos conceptos fundamentales que facilitan la comprensión del tema, el primero de ellos es el de persona mayor $\mathrm{y}$, el segundo, el de envejecimiento saludable activo.

\section{Persona mayor}

Hasta hace poco tiempo había una falta de acuerdo sobre cómo referirse al colectivo de mayores. En Francia y Bélgica se les denominaba "jubilados"; en Dinamarca, Holanda y México la denominación era "ancianos"; "ciudadanos de la tercera edad" en Reino Unido e Irlanda; y "personas mayores" en España, Italia, Portugal, Luxemburgo, Grecia, Alemania y algunos otros países. El concepto de persona mayor ha estado muy vinculado a la edad cronológica o a la edad de jubilación laboral (Causapié, 2011).

En los países desarrollados, y más por un sentido administrativo, se ha determinado que una persona mayor es quien tiene 65 años o más; en otros países como México, se considera a la persona mayor a partir de los 60 años, pero tiene más que ver con la etapa de jubilación que con un concepto social claro.

\section{Envejecimiento saludable activo}

Causapié (2011) señala que la Organización Mundial de la Salud (oms) define el término de envejecimiento activo como el proceso de optimización de las oportunidades de salud, participación y seguridad que viven las personas con el fin de mejorar la calidad de vida a medida que avanzan en su edad. En esta definición se considera que es apremiante promover la participación comunitaria más allá de la edad que la persona tenga y no limitar la autonomía física o la económica.

Los doctores Enrique Vega, asesor regional sobre envejecimiento de la OMS, y Amelia Elisa Ayala, asesora en la Organización Panamericana de la Salud en México (OPS) (Ayala y Vega, 2015), proponen que es necesario hablar del envejecimiento activo, pero es más conveniente que sea tanto saludable, como activo; por lo que en este do- cumento se designa a la condición de vivir la edad adulta en condiciones favorables de salud física, emocional y social como envejecimiento saludable activo.

\section{Aspectos universitarios esenciales al hablar del envejecimiento saludable activo}

Existen dos aspectos fundamentales cuando se habla del envejecimiento saludable activo; el primero es que se debe fortalecer la educación para las personas mayores que tienen experiencia y una condición de reflexión y memoria diferente a las personas más jóvenes, por lo que se deben considerar las buenas prácticas que la psicología educativa sugiere (Gerontagogía). ${ }^{1}$

El segundo es el espacio universitario como escenario de aprendizaje, que debe contar con los elementos necesarios para atender a las personas mayores en los que se sientan cómodos, tomados en cuenta como estudiantes de la institución y, además, en los que se favorezca la convivencia con los estudiantes de distintas edades. Con lo que se espera lograr que su experiencia sirva a los más jóvenes, y que las personas mayores convivan con estudiantes que les aporten ideas diferentes sobre la realidad que en este momento les toca vivir, con el uso de la tecnología, las nuevas dinámicas familiares y sociales.

\section{La educación para personas mayores como una alternativa para promover su envejecimiento saludable activo}

En la actualidad, los programas educativos para personas mayores deben buscar una ampliación de intereses y temáticas que sean propios para dichas personas y que, además, generen conocimiento, lo difundan y participen en aprendizajes autodirigidos y en proyectos de investigación (Causapié, 2011).

La construcción de una sociedad para todas las edades supone un énfasis especial en la promoción de una educación intergeneracional, que

1 Es una nueva disciplina que asume la formación de las personas mayores. Se encarga de desarrollar nuevos modelos de formación de adultos. 
《 Preparar a la población para llegar

a los 60 años o más con la información

y formación adecuada para lograr

una independencia económica, salud física y autoestima alta $\gg$

\section{Conclusiones}

Es necesario desechar la idea de proporcionar una educación a las personas mayores sólo para anticipar su deterioro cognitivo y psicosocial; más bien se les debe brindar una educación permanente y de calidad, como soporte a su desarrollo liberador ante la cambiante sociedad actual, a la cual tienen aún mucho que aportar. En tal sentido, toca a las políticas públicas mantener en alto el debate sobre el futuro del crecimiento poblacional de las personas mayores que, de no tomarse en cuenta en el mediano plazo, puede enfrentar una de las mayores crisis sociales del siglo XXI.

Un enfoque basado en el aprendizaje para la vida requiere integrar la idea central de que las personas mayores participen activamente y tomen decisiones informadas sobre su salud y bienestar, un concepto que también es fundamental para un programa centrado ellas. Las políticas y los programas deben empoderarlas para que contribuyan a la sociedad y sigan siendo miembros activos de sus comunidades durante el mayor tiempo posible, en función de sus capacidades.

Las personas mayores contribuyen al desarrollo social de muchas maneras, por lo que deben ser incluidas en los procesos de desarrollo, lo cual ayuda a fomentar una sociedad más equitativa. Excluirlas de estos procesos, además de socavar su bienestar y sus contribuciones, puede repercutir en el bienestar y en la productividad de otras generaciones.
Una razón más para diseñarles un programa es el aspecto económico, al reducir los gastos asociados con el envejecimiento saludable activo de la población aumentarán al máximo las múltiples contribuciones que realizan las personas mayores, mediante la participación directa en la fuerza de trabajo y bienes que le pueden ofrecer a las generaciones más jóvenes, así como las numerosas prestaciones menos tangibles que hacen a sus familias y comunidades.

De esta manera, las universidades se convierten en un espacio de respuesta a las necesidades de la sociedad para lograr el intercambio de experiencias intergeneracionales y el apoyo al logro del envejecimiento saludable activo.

\section{Fuentes de consulta}

Ayala, E. y Vega, E. (2015). Envejecimiento activo y saludable, visión oms/OPS. Organización Panamericana de la Salud y Organización Mundial de la Salud. Encuentro MéxicoJapón 2015. México: oms/ops.

Causapié, P. (2011). Envejecimiento Activo. Libro Blanco, Ministerio de Sanidad, Política Social e Igualdad. Madrid: IMSERSO.

Consejo Nacional de la Población. (2016). Proyecciones de la población en México 2005- 2050. Distrito Federal: conAPO. Recuperado el 22 de mayo de 2016, en: http://bit. ly/1PGzrWD

Instituto Nacional de Estadística y Geografía. (2015). Encuesta Intercensal. México: INEGI. Latapí, P. (1985). Igualdad, desigualdad y equidad en España y México. Madrid: ICI/COLMEX.

Organización Mundial de la Salud. (2015). Informe mundial sobre el envejecimiento y la salud. Ginebra: OMS.

Portero, F. (1999). La gerontagogía una nueva disciplina. España: Universidad de la Rioja. Recuperado el 24 de febrero de 2017, en: https://dialnet.unirioja.es/descarga/articulo/195859.pdf 